\title{
The relationship between dyslipidemia and disease activity in Iranian population with systemic lupus erythematosus
}

\author{
Sepideh Hajian ${ }^{\mathbb{D}}$, Mohammad Ali Hosseini ${ }^{2,3^{*}}\left(\mathbb{D}\right.$, Mohadaseh Ameri $^{4}$, Sara Khosraviani ${ }^{2}$, Farnaz Tavasoli ${ }^{2}$, \\ Mohammad Ehsan Bayatpoor ${ }^{5}$
}

${ }^{1}$ Department of Nephrology, Velayat Research Center, Qazvin University of Medical Sciences, Qazvin, Iran

${ }^{2}$ Student Research Committee, Qazvin University of Medical Sciences, Qazvin, Iran

${ }^{3}$ Infertility and Reproductive Health Research Center, Shahid Beheshti University of Medical Sciences, Tehran, Iran

${ }^{4}$ Shahid Beheshti University of Medical Sciences, Tehran, Iran

${ }^{5}$ Student Research Committee, Baqiyatallah University of Medical Sciences, Tehran, Iran

\section{A R T I C L E I N F 0}

Article Type:

Original

\section{Article History:}

Received: 6 August 2018

Accepted: 18 October 2018

ePublished: 2 November 2018

\section{Keywords:}

Dyslipidemia

Systemic lupus erythematous

Disease activity

\begin{abstract}
A B S T R A C T
Introduction: In spite of the high prevalence of dyslipidemia in systemic lupus erythematosus (SLE) and its role in patients' cardiovascular and kidney diseases, few studies had been conducted in this regard.

Objectives: We aimed to study the relation between dyslipidemia and disease activity in SLE patients in Iran.

Patients and Methods: This analytical cross-sectional study was conducted on SLE patients. The activity of disease was determined by SLE disease activity index (SLEDAI). The serum level of triglyceride (TG), cholesterol, low-density lipoprotein (LDL-C), and high-density lipoprotein (HDL-C) were measured. The correlation between dyslipidemia and SLE disease activity was assessed.

Results: Around 62 out of 71 patients (87\%) were female (mean age was 34 years). The mean disease duration was 1 year. Around $49 \%$ of patients had active disease (SLEDAI $\geq 6$ ). Proteinuria and nephritis were observed in $18 \%$ and $24 \%$, respectively. About $62 \%$ of patients had at least one abnormality in their lipid profile. High cholesterol $(>200 \mathrm{mg} / \mathrm{dL})$, high TG $(>150 \mathrm{md} / \mathrm{dL})$, high LDL-C $(>130 \mathrm{mg} / \mathrm{dL})$ and low HDL-C $(<50 \mathrm{mg} / \mathrm{dL}$ in females and $<40$ $\mathrm{md} / \mathrm{dL}$ in males) levels were observed in $25 \%, 42 \%, 20 \%$ and $49 \%$ of patients, respectively. Patients with active disease had lower age and shorter disease duration in comparison to the others $(\mathrm{P}<0.05)$. There were no differences in gender and weight between patients in active and inactive phases $(P>0.05)$. Patients with active disease had higher serum cholesterol, TG and LDL-C levels and lower levels of serum HDL-C.

Conclusion: It seems that there is a relationship between disease activity and lipid profile abnormalities in SLE patients.
\end{abstract}

Implication for health policy/practice/research/medical education:

In a study on 71 SLE patients, we concluded that dyslipidemia is commonly present in SLE patients in the Iranian population. SLE patients with active disease have higher levels of cholesterol, TG, LDL-C and lower range of HDL-C than patients with inactive disease.

Please cite this paper as: B. The relationship between dyslipidemia and disease activity in Iranian population with systemic lupus erythematosus. J Nephropharmacol. 2019;8(1):e06. DOI: 10.15171/npj.2019.06

Introduction

Systemic lupus erythematosus (SLE) is a chronic autoimmune disease of unknown etiology which can affect many organs of the body and has a broad spectrum of manifestations (1). Lipid profile abnormalities are one of the most important and effective risk factors for cardiovascular events which is an important factor for morbidity and mortality in patients with SLE (2). It has already been identified that cardiovascular disease in SLE patients can be related to routine risk factors such as hypertension, obesity, dyslipidemia and diabetes. However, other risk factors such as constant activation of immune 
system and a continuous inflammatory process such as atherogenic factors, inflammatory markers specially C-reactive protein (CRP), elevated homocysteine, high levels of tumor necrosis factor (TNF- $\alpha$ ), lower folate levels, numerous autoantibodies like anti-phospholipid antibody (APA), anti-lipoprotein lipase (ALPL) $(3,4)$ may be responsible for cardiovascular events. Dyslipidemia includes hypertriglyceridemia, hypercholesterolemia, increase of low-density lipoprotein (LDL-C), and decrease of high-density lipoprotein (HDL-C) which, as suggested in some studies, is related to lupus disease activity (5).

It has been reported that in patients with SLE, activity of lipoprotein lipase (LPL), compared to that of normal people, decreases to $50 \%$ (6). This enzyme plays an important role in purifying VLDL-rich triglycerides (verylow density lipoprotein). It is expected that the level of triglycerides (TGs) will increase in these patients because of decreasing the activity of this enzyme. In addition, HDL-C is a vascular protector factor which triggers the movement of cholesterol from the atherosclerotic vessels to the liver. Additionally its anti-inflammatory function inhibits the adhesion of endothelial cells (7). Therefore, it seems that HDL-C reduction and lupus activity are interconnected.

\section{Objectives}

Due to the high prevalence of lipid profile abnormalities (dyslipidemia) in SLE patients and its role in cardiovascular events, we aimed to evaluate the prevalence of dyslipidemia in SLE patients and its relation with disease activity.

\section{Patients and Methods}

\section{Study patients}

This analytical descriptive study was conducted on SLE patients based on American College of Rheumatology (ACR) attending to Hasheminejad and Velayat hospitals, Iran (2014 to 2016). Around 71 patients were enrolled in this study. The exclusion criteria were patients with diabetes mellitus, abnormal thyroid function tests, consuming statins and oral contraceptive pills, pregnant women, febrile illness, or familial dyslipidemia. The demographic information (age, sex, and weight), renal condition (proteinuria and nephritis), laboratory tests (anti-dsDNA, anti-cardiolipin antibodies [ACLA] and complement levels). Consumption of prednisolone, chloroquine and their doses were recorded in a checklist. Additionally SLE disease activity index (SLEDAI) for diagnosis and grading the disease was applied. According to the SLEDAI score, six or higher was considered as an active disease and less than six was considered as an inactive disease. Then cholesterol, TG, LDL-C and HDL-C were assessed and compared between active and inactive groups.

\section{Definitions}

High cholesterol level was defined as cholesterol level higher than $200 \mathrm{mg} / \mathrm{dL}$. High triglyceride level was defined as TG higher than $150 \mathrm{mg} / \mathrm{dL}$, while high LDL-C level was defined as, LDL-C higher than $130 \mathrm{mg} / \mathrm{dL}$. Accordingly, low HDL-C level was defined as HDL-C lower than 50 $\mathrm{mg} / \mathrm{dL}$ in women and lower than $40 \mathrm{mg} / \mathrm{dL}$ in men.

\section{Ethical issues}

The research followed the tenets of the Declaration of Helsinki. Before the study, written informed consent was obtained from all participants who participated in the study. All information of individuals was coded and kept confidential.

\section{Statistical analysis}

The sample size was calculated by the previous reported data. All data analysis was performed using SPSS version 13. Qualitative variables were described as frequency and relative frequency (\%), quantitative variables were described as median (IQR, range). To compare quantitative variables between two groups with active and inactive disease we used independent $t$ test and for quantitative variables between two groups we used chi-square, MannWhitney $U$ and Fisher's exact tests.

To eliminate the bias of hydroxychloroquine and prednisolone on lipid changes between two groups we used two-layered regression analysis. In first layer, hydroxychloroquine (with three levels of non-intake, 200 $\mathrm{mg} / \mathrm{d}$ and $400 \mathrm{mg} / \mathrm{d}$ doses) and prednisolone (with three levels of non-intake, lower than $10 \mathrm{mg} / \mathrm{d}$, equal or higher than $10 \mathrm{mg} / \mathrm{d}$ ) were enrolled with ENTER method. In both cases, non-consumption was selected as the basis for the comparison. In second layer, cholesterol, TG, HDL-C and LDL-C enrolled with FORWARD CONDITION method [each of them was bi-state (normal or abnormal)]. The basis of comparison in these variables was normal state. Values of $P<0.05$ were regarded as statistically significant.

\section{Results}

In total, 71 patients were included in this study. Around $62(87 \%)$ of patients were female. Patients had median (IQR) age of 34 (15- 71) years with median body mass index (BMI) of the patients was $26.5(35-19.5) \mathrm{kg} / \mathrm{m}^{2}$. The average of SLE duration in patients was $2.4 \pm 2.8$ years and $21 \%$ of patients had been diagnosed for less than 1 month. Around $49 \%$ of patients had active disease (SLE DAI score $\geq 6$ ) while $51 \%$ of them had inactive disease (SLE DAI score $<6)$. About 13 patients $(18 \%)$ had proteinuria and 17 patients (24\%) had nephritis. Around 42 patients (59\%) had abnormal dsDNA (dsDNA $\geq 20 \mathrm{ng}$ ), complement was decreased in 32 patients (45\%) and ACLA was positive in 8 patients (11\%). About 37 patients (52\%) were consuming hydroxychloroquine and also 41 patients (58\%) were taking prednisolone (Table 1).

\section{Lipid profile}

Forty-four patients (62\%) had at least one lipid profile 
Table 1. Baseline characteristics, laboratory tests and drug consumption of the patients

\begin{tabular}{|c|c|c|c|}
\hline & $\begin{array}{l}\text { Active } \\
\text { group }\end{array}$ & $\begin{array}{l}\text { Inactive } \\
\text { group }\end{array}$ & $P$ value \\
\hline \multicolumn{4}{|l|}{ Gender } \\
\hline Male & $4(11 \%)$ & $5(14 \%)$ & 1.000 \\
\hline Female & $31(89 \%)$ & $31(86 \%)$ & 1.000 \\
\hline Age (year), median (IQR) & $31 \pm 11$ & $38 \pm 12$ & 0.091 \\
\hline BMI, median (IQR) & 26.2 & 26.8 & $>0.005$ \\
\hline Proteinuria, prevalence (\%) & $13(37 \%)$ & $0(0 \%)$ & $<0.001^{*}$ \\
\hline Nephritis, prevalence (\%) & $16(46 \%)$ & $1(3 \%)$ & $<0.001^{*}$ \\
\hline High anti-dsDNA & $36(100 \%)$ & $6(17 \%)$ & $<0.001^{*}$ \\
\hline Decreased complement & 27 (77\%) & $5(14 \%)$ & $<0.001^{*}$ \\
\hline Positive ACLA & $6(17 \%)$ & $2(6 \%)$ & 0.151 \\
\hline Cholesterol (mg/dL), median (IQR) & $206 \pm 70$ & $145 \pm 37$ & $<0.001^{*}$ \\
\hline TG (mg/dL), median (IQR) (range) & $238 \pm 167$ & $122 \pm 47$ & $<0.001^{*}$ \\
\hline LDL (mg/dL), median (IQR) (range) & $113 \pm 46$ & $77 \pm 34$ & $<0.001^{*}$ \\
\hline $\mathrm{HDL}$ (mg/dL), median (IQR) (range) & $40 \pm 22$ & $50 \pm 12$ & $0.018^{*}$ \\
\hline High cholesterol & $15(43 \%)$ & $3(8 \%)$ & $0.001 *$ \\
\hline High TG & $21(60 \%)$ & $9(25 \%)$ & $0.003 *$ \\
\hline High LDL & $23(66 \%)$ & $12(33 \%)$ & $0.006^{*}$ \\
\hline Low HDL & $11(31 \%)$ & $3(8 \%)$ & $0.014 *$ \\
\hline HCQ non-intake & $19(54 \%)$ & $15(42 \%)$ & 0.285 \\
\hline HCQ 200 mg dose & $6(17 \%)$ & $12(23 \%)$ & 0.285 \\
\hline HCQ 400 mg dose & $10(29 \%)$ & $9(25 \%)$ & 0.285 \\
\hline Prednisolone non-intake & $14(40 \%)$ & $16(44 \%)$ & $0.026^{*}$ \\
\hline Prednisolone $<10 \mathrm{mg}$ & $7(20 \%)$ & $15(42 \%)$ & $0.026 *$ \\
\hline Prednisolone $\geq 10 \mathrm{mg}$ & $14(40 \%)$ & $5(14 \%)$ & $0.026 *$ \\
\hline
\end{tabular}

ACLA: anti cardiolipin antibody, TG: triglyceride, LDL:Iow density lipoprotein, HDL: high density lipoprotein, HCQ: Hydroxichloroquine. $* P \leq 0.05$ is considered as significant.

abnormality. Figure 1 indicates abnormal lipid profile frequencies. High cholesterol (>200 mg/dL) in $25 \%$, high TG $(>150 \mathrm{mg} / \mathrm{dL})$ in $42 \%$, high LDL-C $(>130 \mathrm{mg} / \mathrm{dL})$ in $20 \%$ and low HDL-C $(<50 \mathrm{mg} / \mathrm{dL}$ in women and $<40 \mathrm{mg} /$ $\mathrm{dL}$ in men) was observed in $49 \%$ of patients ( $22 \%$ in men and $53 \%$ in women) (Figure 1).There were no significant differences between gender and weight in the two groups $(P>0.05)$. The active group had significantly lower age than inactive group $(P<0.05)$. There was no significant association between gender and dyslipidemia $(P>0.05)$. BMI had no significant correlation with dyslipidemia

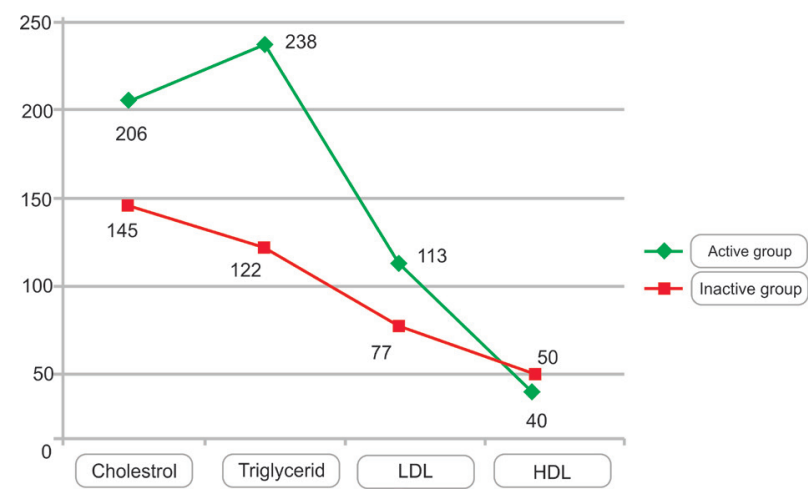

Figure 1. Frequency of lipid profile abnormalities in patients.
$(P>0.05)$. In patients with lower age, values of cholesterol and LDL-C were higher $(P<0.001)$. SLE duration was significantly lower in active group $(P<0.001$, MannWhitney $\mathrm{U}$ test). Lower duration of SLE was associated with dyslipidemia $(P<0.05)$. Proteinuria and nephritis were significantly higher in patients with active disease $\left(P<0.001, \quad \chi^{2}\right.$ test $)$. Accordingly the manifestations of active group of SLE patients were lupus nephritis (46\%), arthritis (46\%), encephalitis (4\%) and serositis (4\%). Lupus nephritis was significantly associated with hypercholesterolemia, high LDL-C level and low HDL-C level $(P<0.001)$. Arthritis had also a significant correlation with hypertriglyceridemia $(P<0.001)$. Anti-dsDNA in patients with active disease was significantly higher than inactive group $(P<0.001)$. The prevalence ACLA had no significant difference between two groups $(P=0.151$, Fisher's exact test). Furthermore, no significant differences of taking of hydroxychloroquine between active and inactive groups were seen $(P=0.285)$. However, patients with active disease had been consuming significantly more prednisolone with high dose $\left(P<0.05, \chi^{2}\right)$. Patients with active disease had higher range of cholesterol, TG and LDL than inactive group $(P<0.001, t$ test) (Figure 2$)$.

In two layered regression analysis, chance of having active disease in patients with high cholesterol was more than six times (OR=6.6, CI 95\% 1.4-30.1). Additionally chance of having active disease in patients with high cholesterol was more than five times, in patients consuming prednisolone, more than $10 \mathrm{mg} / \mathrm{d}(\mathrm{OR}=5.6$, CI $95 \%=1.3-24.1)$ and more than three times in patients with low HDL-C $(\mathrm{OR}=3.4$, CI $95 \%=1.0-11.5)$ (Table 2).

\section{Discussion}

Our study showed that nearly two-thirds of patients with SLE had abnormal lipid profiles. The most common abnormality was high triglyceride and low HDL-C serum concentrations. Dyslipidemia is common in SLE patients. Various studies have shown different prevalence's of dyslipidemia (63\% to $75 \%$ ) in patients with SLE which is

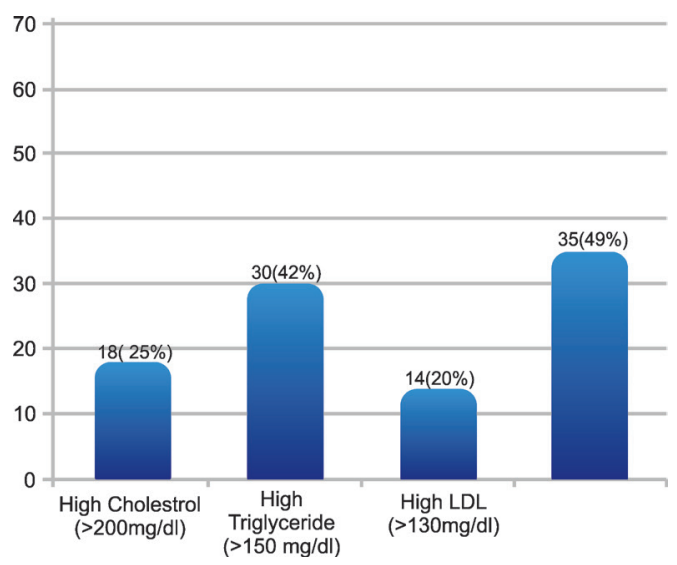

Figure 2. Comparison of the lipid profile in the active group and the inactive group 
Table 2. The results of logistic regression analysis for having chance of active disease in patients

\begin{tabular}{|c|c|c|c|c|c|c|c|c|}
\hline & \multirow{2}{*}{ B } & \multirow{2}{*}{ SE } & \multirow{2}{*}{ Wald } & \multirow{2}{*}{$d f$} & \multirow{2}{*}{$P$ value } & \multirow{2}{*}{$\operatorname{Exp}(B)$} & \multicolumn{2}{|c|}{$95 \% \mathrm{Cl}$ for $\operatorname{EXP}(\mathrm{B})$} \\
\hline & & & & & & & Lower & Upper \\
\hline Prednisolone_Dose_None & & & 6.597 & 2 & 0.037 & & & \\
\hline Prednisolone_Dose_<10 & -0.079 & 0.719 & 0.012 & 1 & 0.913 & 0.924 & 0.226 & 3.780 \\
\hline Prednisolone_Dose_>=10 & 1.722 & 0.746 & 5.333 & 1 & 0.021 & 5.596 & 1.298 & 24.129 \\
\hline Hydroxichloroquine_Dose_None & & & 1.644 & 2 & 0.439 & & & \\
\hline Hydroxichloroquine_Dose_200 & -0.966 & 0.760 & 1.617 & 1 & 0.204 & 0.380 & 0.086 & 1.688 \\
\hline Hydroxichloroquine_Dose_400 & -0.369 & 0.710 & 0.270 & 1 & 0.603 & 0.691 & 0.172 & 2.779 \\
\hline Chol_High & 1.887 & 0.774 & 5.947 & 1 & 0.015 & 6.601 & 1.448 & 30.083 \\
\hline HDL_Low & 1.230 & 0.619 & 3.949 & 1 & 0.047 & 3.420 & 1.017 & 11.500 \\
\hline Constant & -1.147 & 0.620 & 3.416 & 1 & 0.065 & 0.318 & & \\
\hline
\end{tabular}

in line with our findings (8-11). However, high cholesterol and high LDL-C are also reported in a variety of studies (12). Taking of steroid is a risk factor for dyslipidemia, however, using hydroxychloroquine has protective effect (13). Lipid profile changes are independently associated cardiovascular events and disease activity and also dyslipoproteinemia pattern in active patients may also be associated with autoimmune mechanisms (4). Meanwhile, low prednisolone dose seems to improve the level of abnormal lipids, but high doses have an adverse effect on lipids. Antimalarial agents may have a beneficial effect on lipid profiles due to decrease of disease activity. The present study showed that SLE patients consuming high doses prednisolone, patients with high levels of cholesterol, and also patients with low levels of HDL-C have the greater chance of obtaining active disease (14). Indeed, lipid profile in patients with SLE is a result of interactions of the manifestation of disease activity, autoimmune and inflammatory mechanisms, prednisolonetherapy and other drugs such as antimalarial agents $(11,15)$.

Leong et al evaluated lipid profile and its risk factors in 100 SLE patients. The results of their study showed that $73 \%$ of patients had abnormal lipid profile. Abnormal levels of cholesterol, triglyceride, LDL-C and HDL-C were associated with renal involvement and high doses of corticosteroids (more than $30 \mathrm{mg}$ prednisolone per day). HDL-C levels were significantly low in patients with high corticosteroid doses. In patients with active disease, but without kidney disease, lipid abnormalities were not observed. Finally, they concluded that lipid profile abnormalities are common in patients with lupus under treatment of high doses of steroids or who have nephrotic syndrome (16). However, Kakati et al evaluated the prevalence of lipid profile abnormalities in SLE patients and the related risk factors. They found abnormal lipid profile is common in SLE patients. Nephropathy, especially with proteinuria in nephrotic range and taking of steroids, are the major risk factors for dyslipidemia (17).

Svenungsson et al examined the relationship between lipid levels with disease activity, clinical features and serum TNF- $\alpha$ levels and its soluble type1 and II receptors in patients with SLE. The results showed that levels of triglyceride were associated with TNF- $\alpha$, disease activity and type II receptors. The level of HDL-C was inversely related to disease activity, TNF- $\alpha$ activity and also its soluble type II receptors. High levels of triglycerides, total cholesterol, TNF- $\alpha$ and its receptors (type I and II) were associated with nephritis and arterial diseases. Finally, it is concluded that dyslipoproteinemia with high triglycerides and low HDL-C levels is associated with disease activity, while the results were similar to our findings (18). In a study in 2005 the prevalence of dyslipidemia in SLE patients and the effect of various factors were measured. They detected that less than three years duration of the disease is strongly associated with dyslipidemia. (19). Furthermore, in a study in 2007, lipid profile and its relation with anti-dsDNA in the duration of disease activity was evaluated. The results showed that patients with active disease, had higher levels of triglyceride and VLDL and lower levels of HDL-C than the patients with inactive disease. Moreover, the levels of triglyceride and VLDL was higher in patients with anti-dsDNA while, HDL-C levels had no significant differences. Finally, it was indicated that this type of dyslipoproteinemia in patients with active disease may be interconnected with autoimmune mechanisms, particularly with antidsDNA antibodies (20). Likewise, in a study in 2007, the relationship between lipid profile and inflammatory markers in patients with SLE was assessed. ESR, CRP, insulin, IL-6 and TNF- $\alpha$ were measured. The results showed that lower levels of HDL-C are associated with high ESR, IL-6 and disease activity. Additionally, higher levels of triglyceride were associated with higher levels of CRP. Finally, it was mentioned that changes in the lipid profile are independently associated with high inflammatory mediators and disease activity (21). Similarly in a study in Spain, lipid profile among SLE patients was evaluated according to disease activity. They indicated that there is a higher risk of atherosclerosis phenomena in SLE patients during flare than during clinical remission (22). Hence, in SLE patients there is higher risk for atherosclerosis in flareup. Therefore, it is required for every patient according to 
their circumstances, lipid profile, disease activity and their drug intake, for prevention of cardiovascular accidents, lipid profile to be regularly checked and if necessary, be treated appropriately.

\section{Conclusion}

This study demonstrated that dyslipidemia is commonly present in Iranian SLE patients. Patients with active disease have higher levels of cholesterol, TG, LDL-C and lower range of HDL-C than inactive disease. Therefore patients with active disease need necessary measures to prevent cardiovascular complications of dyslipidemia. In patients with active disease, in addition to the drugs used to reduce the severity of the disease, if necessary, treatment of dyslipidemia should be considered. Prospective studies are needed to be designed to investigate the causal relation between dyslipidemia and disease activity in order to determine the possible mechanisms of their impact.

\section{Limitation of the study}

One limitation of the present study was the small sample size. Despite of two years duration of the study, due to the low cases with inclusion criteria at Hasheminejad and Velayat hospitals (Tehran and Qazin; Iran), the study was conducted on 72 patients. Furthermore, refusal to consent was the second limitation of the study.

\section{Authors' contribution}

$\mathrm{SH}, \mathrm{MAH}$ and SK; project development, data collection and data analysis. FT and MAH; writing the manuscript. MA and FT; editing the manuscript. $\mathrm{SH}, \mathrm{MAH}, \mathrm{MA}$ and MEB; final check and edit of paper. All authors read and signed the final version of the manuscript.

\section{Conflicts of interest}

The authors declare no conflict of interest.

\section{Ethical considerations}

Ethical issues (including plagiarism, data fabrication, double publication) have been completely observed by the authors.

\section{Funding/Support}

There is no source of support, conflicts of interest and permission.

\section{References}

1. Petri M, Perez-Gutthann S, Spence D, Hochberg MC. Risk factors for coronary artery disease in patients with systemic lupus erythematosus. Am J Med Sci. 1992;93:513-9.

2. Shoenfeld Y, Gerli R, Doria A, Matsuura E, Cerinic MM, Ronda N, et al. Accelerated atherosclerosis in autoimmune rheumatic diseases. Circulation. 2005;112:3337-47.

3. Ehnholm C, Aho K, Huttunen JK, Kostiainen E, Mattila $\mathrm{K}$, Pakkarainen J, et al. Effect of interferon on plasma lipoproteins and on the activity of postheparin plasma lipases. Arterioscler Thromb Vasc Biol. 1982;2:68-73.

4. Deckelbaum RJ, Granot E, Oschry Y, Rose L, Eisenberg S. Plasma triglyceride determines structure-composition in low and high density lipoproteins. Arterioscler Thromb Vasc Biol. 1984;4:225-31.

5. Svenungsson E, Jensen-Urstad K, Heimbürger M, Silveira A, Hamsten A, de Faire U, et al. Risk factors for cardiovascular disease in systemic lupus erythematosus. Circulation. 2001;104:1887-93.

6. Borba EF, Carvalho JF, Bonfá E. Mechanisms of dyslipoproteinemias in systemic lupus erythematosus. J Immunol Res. 2006;13:203-8.

7. Lee A, Godfrey T, Rowley K, Karschimkus C, Dragicevic G, Romas E, et al. Traditional risk factor assessment does not capture the extent of cardiovascular risk in systemic lupus erythematosus. Intern Med J. 2006;36:237-43.

8. Glueck CJ, Levy RI, Glueck HI, Gralnick HR, Greten H, Fredrickson DS. Acquired type I hyperlipoproteinemia with systemic lupus erythematosus, dysglobulinemia and heparin resistance. Am J Med. 1969;47:318-24.

9. Borba E, Bonfa E. Dyslipoproteinemias in systemic lupus erythematosus: influence of disease, activity, and anticardiolipin antibodies. Lupus. 1997;6:533-9.

10. Jacobs Jr DR, Mebane IL, Bangdiwala SI, Criqui MH, Tyroler HA, PROGRAM LRC. High density lipoprotein cholesterol as a predictor of cardiovascular disease mortality in men and women: the follow-up study of the Lipid Research Clinics Prevalence Study. Am J Epidemiol. 1990;131:32-47.

11. Dinu A, Merrill J, Shen C, Antonov I, Myones B, Lahita R. Fequency of antibodies to the cholesterol transport protein apolipoprotein A1 in patients with SLE. Lupus. 1998;7:35560.

12. Asanuma Y, Chung CP, Oeser A, Shintani A, Stanley E, Raggi P, et al. Increased concentration of proatherogenic inflammatory cytokines in systemic lupus erythematosus: relationship to cardiovascular risk factors. J Rheumatol. 2006;33:539-45.

13. Lahita R, Rivkin E, Cavanagh Ms I, Romano P. Low levels of total cholesterol, high-density lipoprotein, and apolipoprotein A1 in association with anticardiolipin antibodies in patients with systemic lupus erythematosus. Arthritis Rheum. 1993;36:1566-74.

14. Frostegård J, Svenungsson E, Wu R, Gunnarsson I, Lundberg IE, Klareskog L, et al. Lipid peroxidation is enhanced in patients with systemic lupus erythematosus and is associated with arterial and renal disease manifestations. Arthritis Rheum. 2005;52:192-200.

15. McMahon M, Grossman J, FitzGerald J, Dahlin-Lee E, Wallace DJ, Thong BY, et al. Proinflammatory high-density lipoprotein as a biomarker for atherosclerosis in patients with systemic lupus erythematosus and rheumatoid arthritis. Arthritis Rheum. 2006;54:2541-9.

16. Leong KH, Koh E, Feng P, Boey M. Lipid profiles in patients with systemic lupus erythematosus. J Rheumatol. 1994;21:1264-7.

17. Kakati S, Doley B, Devee A, Dihingia P, Sarma P. Serum lipid profiles in patients with systemic lupus erythematosus. J Indian Rheumatol Assoc. 2003;11:5-7.

18. Svenungsson E, Gunnarsson I, Fei GZ, Lundberg IE, Klareskog L, Frostegård J. Elevated triglycerides and low 
levels of high-density lipoprotein as markers of disease activity in association with up-regulation of the tumor necrosis factor $\alpha /$ tumor necrosis factor receptor system in systemic lupus erythematosus. Arthritis Rheum. 2003;48:2533-40.

19. Wijaya LK, Kasjmir YI, Sukmana N, Subekti I, Prihartono J. The proportion of dyslipidemia in systemic lupus erythematosus patient and distribution of correlated factors. Acta Med Indones. 2005;37:132-44.

20. Kashef S, Ghaedian MM, Rajaee A, Ghaderi A. Dyslipoproteinemia during the active course of systemic lupus erythematosus in association with anti-double- stranded DNA (anti-dsDNA) antibodies. Rheumatol Int. 2007;27:235-41.

21. Chung CP, Oeser A, Solus J, Avalos I, Gebretsadik T, Shintani A, et al. Inflammatory mechanisms affecting the lipid profile in patients with systemic lupus erythematosus. J Rheumatol. 2007;34:1849-54.

22. Urquizu-Padilla $M$, Balada E, Chacon P, Pérez EH, Vilardell-Tarrés M, Ordi-Ros J. Changes in lipid profile between flare and remission of patients with systemic lupus erythematosus: a prospective study. J Rheumatol. 2009;36:1639-45.

Copyright $\odot 2019$ The Author (s); Published by Society of Diabetic Nephropathy Prevention. This is an open-access article distributed under the terms of the Creative Commons Attribution License (http://creativecommons.org/licenses/by/4.0), which permits unrestricted use, distribution, and reproduction in any medium, provided the original work is properly cited. 